\title{
Clinical and Emergent Biomarkers and Their Relationship to the Prognosis of Ovarian Cancer
}

\author{
Aminah Jatoi $^{a} \quad$ Robert A. Vierkant $^{b}$ Kieran M. Hawthorne ${ }^{b}$ Matthew S. Block ${ }^{a}$ \\ Susan J. Ramus ${ }^{d}$ Nicholas B. Larson ${ }^{b}$ Brooke L. Fridley ${ }^{e}$ Ellen L. Goode ${ }^{c}$ \\ Departments of a Oncology, ${ }^{\mathrm{b}}$ Biomedical Statistics and Informatics, and ${ }^{\mathrm{c} H e a l t h}$ Sciences Research, Mayo Clinic, \\ Rochester, Minn., ${ }^{d}$ Department of Preventive Medicine, Keck School of Medicine at the University of Southern \\ California, Los Angeles, Calif., and e Department of Biostatistics, University of Kansas, Kansas City, Kans., USA
}

\section{Key Words \\ Ovarian cancer $\cdot$ Modeling $\cdot$ Prognosis $\cdot$ Interactions}

\begin{abstract}
Objective: Ovarian cancer is the most lethal gynecological malignancy, but information relevant to prognosis and outcomes remain unknown. Here, we used statistical methods to focus specifically on interactions between candidate prognostic variables. Methods and Results: Univariate, multivariate, and elastic net modeling of 42 variables were applied to a cohort of 542 ovarian cancer patients with $393 \mathrm{ep}$ isodes of cancer recurrence/death. In univariate analyses, overexpression of TFF3, MDM2, and p53 were associated with improved recurrence-free survival. In multivariate analyses adjusted for age, histology, stage, grade, ascites, and residual disease, overexpression of $P R$ appeared to provide a protective effect [hazard ratio for $>50 \%$ of cells positive, 0.64 (95\% confidence interval $0.44-0.94$ ) compared to $<1 \%]$, and TFF3 showed a nonlinear association. Importantly, we observed no interactions among variables. However, patients with tumors with moderate TFF3 expression were at a
\end{abstract}

\section{KARGER}

E-Mail karger@karger.com www.karger.com/ocl marginally increased risk of recurrence, and patients with tumors with high expression were at a similar to slightly lower risk, compared to those with tumors with no TFF3 expression. Conclusions: Although no interactions among variables were observed, this study provides important precedent for seeking interactions between clinical and tumor variables in future studies.

(c) 2016 S. Karger AG, Basel

\section{Introduction}

Ovarian cancer is the most lethal gynecological malignancy [1]. Although previous studies have identified tumor characteristics associated with the development of ovarian cancer, fewer studies have explored the prognostic significance of such factors within the context of multivariate models that include clinical characteristics and potential interactions among all such variables.

To our knowledge, an appraisal of interactions among previously reported clinical and tumor-related prognostic factors has not yet been undertaken in ovarian cancer.
(C) 2016 S. Karger AG, Basel

0030-2414/16/0902-0059\$39.50/0
Dr. Aminah Jatoi

Department of Oncology, Mayo Clinic, Rochester 200 First Street SW

Rochester, MN 55905 (USA)

E-Mail jatoi.aminah@mayo.edu 
The inability of standard analysis tools to model large sets of predictors has precluded such efforts. Yet, such interactions may exist and could conceivably enhance the accuracy of extant prognostic information. As an example, paclitaxel, an agent commonly used to treat ovarian cancer, appears to induce tumor expression of the myeloid differentiation primary response gene ( $M y D 88)$, which, in turn, appears to lead to chemotherapy resistance $[2,3]$. Estrogen and progesterone appear to inhibit $M y D 88$, thereby illustrating how clinical and tumorrelated factors - chemotherapy, $M y D 88$, and estrogen/ progesterone - might together plausibly give rise to interactions that modify clinical outcomes. Determining the existence and extent of such putative interactions could enhance our ability to predict cancer recurrence with greater accuracy.

This study was undertaken with a twofold purpose. First, we examined prognostic associations among tumor markers. These markers included TFF3, WT1, p16, MDM2, and p53 and were chosen because of no well-established interactions. The rationale is that this lack of established interactions would enhance the novelty of findings that may emerge from our second purpose. Second, we examined associations of ovarian cancer survival with pairwise and higher-order interactions of clinical factors and tumor expression variables in a series of ovarian cancer cases using contemporary elastic net and classification tree analysis tools in an effort to capture interactions among variables.

\section{Methods}

Overview of Patients and Data Sources

This study was conducted at the Mayo Clinic after Institutional Review Board approval. It focused on women 18 years of age or older diagnosed with pathologically confirmed primary invasive epithelial ovarian cancer, fallopian tube cancer, or primary peritoneal cancer. Because these three malignancies are treated in a uniform manner, herein, we refer to all three as 'epithelial ovarian cancer'.

Data were acquired from multiple sources: (1) patient-completed risk factor questionnaires; (2) formalin-fixed paraffin-embedded tumor tissue, and (3) patients' medical records and additional pathological review with abstracted details such as tumor histology, type of surgery, and type of chemotherapy. Information on cancer recurrence was updated from the Mayo Clinic electronic medical record and defined based on the date of starting cancer treatment for recurrent cancer. A follow-up mailed questionnaire also elicited information from patients about whether their cancer had recurred with subsequent medical record confirmation when available.

\section{Clinical Factors}

Clinical factors of interest consisted of patient age at cancer diagnosis, tumor histology, tumor stage, tumor grade, whether or not ascites was present at surgery, and extent of postoperative residual disease. In addition, patient age at menarche, use of oral contraceptives, parity, education level, and smoking status were also explored. All these clinical variables were chosen because previous studies had suggested prognostic relevance [4-6]. Of note, the majority of patients received cancer therapy in keeping with established guidelines with debulking surgery followed by 18 weeks of platinum-based chemotherapy.

\section{Potential Tumor Prognostic Factors}

Tumor-related factors were chosen either because a specific variable had previously been demonstrated to have a prognostic association or because of a purported mechanism-based role in ovarian cancer development or tumor growth. These factors include the following: ARID1A, $\beta 2$ defensin, CD8, CD68, DKK1, ER, fibrinogen, FOLR1, gp96, heparan sulfate, heparanase, high-mobility group box 1, HNF1B, hsp60, hsp70, I $\mathrm{B} \alpha, \mathrm{I} \kappa \mathrm{B} \beta, \mathrm{MDM} 2$, MMP9, MyD88, p16, p50, p53, p65, phospho-IкBa, phospho-p65, PR, TFF3, TLR4, vimentin, and WT1 [7-10]. It should be noted that the main emphasis of this paper was on the statistical methodology that centered on identifying and understanding variable interactions; for this reason, variables were not derived from wellestablished pathways with already well-demonstrated interactions between variables.

Tissue microarrays were created from formalin-fixed, paraffinembedded tumors. Slides were immunostained with primary antibodies that recognized the proteins described above after optimization of staining conditions on positive control tissues. Slides were then scored by two reviewers. Details are available upon request. The strongest protein expression over multiple cores was used for scoring, and discrepancies were resolved by a gynecologic pathologist.

\section{Statistical Methods}

Imputation of Missing Values

Prior to analysis, imputation of missing clinical, lifestyle, and tumor expression values was performed using the MICE package in $\mathrm{R}$ 2.15.0. All variables with at least one missing value were imputed using a regression model that included all other independent variables as predictors. Dichotomous variables (high vs. low tumor grade, presence of ascites, ever used oral contraceptives, and ever smoked) were imputed using a binary logistic regression model, multilevel nominal characteristics (parity/age at first birth, education, and age at menarche) using unordered multinomial logistic regression, and ordinal characteristics (extent of residual disease and each of the tumor expression variables) using proportional odds logistic regression. Imputation was performed five times, resulting in five complete datasets.

\section{Univariate and Multivariate Analyses}

Cox proportional hazards regression was used to calculate survival estimates, with left truncation to account for delayed study enrollment and right censoring at 10 years to minimize competing causes of death. The main effect associations for each of the 42 potential prognostic variables were first examined in univariate analyses. Separate models were fit for each of the five complete imputed datasets, and unified parameter estimates and 95\% confi- 
dence intervals (CI) were calculated with standard multiple imputation methods [11].

Next, a multivariate Cox regression model was fit to examine independent associations of clinical, lifestyle, and tumor expression variables with recurrence-free survival. The large number of predictors (42 variables with 51 corresponding degrees of freedom) relative to the sample size of 542 subjects prohibited the simultaneous inclusion of all variables in one model, and the introduction of multiple imputed datasets dramatically increased the complexity of common variable selection approaches such as stepwise regression. To overcome this, the Group LASSO (GL) regularization models were used to select variables to enter the final multivariate model [12]. Briefly, LASSO is a statistical regularization method that imposes penalty terms to regression-based parameter estimates to prevent overfitting of a model [13]. These penalty terms serve the function of shrinking the parameter estimates toward zero (the null hypothesis). LASSO differs from ridge regression, another statistical regularization method, in that parameter estimates based on the former can be shrunk completely back to zero, whereas estimates from the latter will always be nonzero. This adds a variable selection component to LASSO: estimates that are shrunk all the way to zero are effectively removed from the model. Crossvalidation is used to tune and optimize the LASSO penalty terms. GL is an extension of LASSO that allows a single penalty term to be simultaneously imposed on the n-1 parameter estimates that compose a given n-level nominal categorical variable, thus preserving the internal structure of that variable. Cox regression-based GL was carried out on each of the five imputed complete datasets using the analysis tool SGL. Variables that were retained in at least four of the five imputed datasets were considered predictive of recurrence-free or overall survival and were included in one final traditional (that is, non-LASSO-based) Cox regression model to aid with interpretation of the parameter estimates. The following six variables were also included in this final model due to their commonly recognized effect on prognosis: age, stage, grade, histology, presence of ascites, and extent of residual disease. Unified parameter estimates and corresponding 95\% CI were again calculated using standard multiple imputation analysis methods.

\section{Interaction Testing}

Pairwise interactions were examined for potential prognostic variables with respect to recurrence-free survival to assess the existence of effect modification. Interactions for each of the 861 possible variable pairs ( 42 choose 2 ) were examined separately by fitting main effects and interactions and testing the statistical significance of the interactions. Due to sparsity of data for some histologic categories, mucinous, clear-cell, and other histologies were combined into one group.

Elastic net survival regression methods were used to simultaneously examine the 861 paired interaction effects [14]. Briefly, elastic net is a family of regularization models that applies penalty functions that are a linear combination of LASSO and ridge regression. These functions can range from 0 to 1 , with those closer to 1 behaving more like LASSO in that fewer variables are retained in the final model, and those closer to 0 behaving more like ridge regression in that more variables are retained. A series of elastic net models were run on each of the five imputed datasets, varying the penalty function from 0.1 to 1.0 . All 42 main ef- fect terms were initially forced into the model and only the interaction terms were shrunk in an effort to retain the structural hierarchy of interaction models. A secondary two-step approach was applied because of concerns that simultaneous inclusion of all prognostic variables would result in an overparameterized model. A model that allowed all main effects and interactions to be shrunk or eliminated was fit. All retained as either a main effect or interaction term were included as main effects in a subsequent model. This initial approach was then applied to a smaller model with a forced reduction in the reduced number of main effect terms and with allowance for their corresponding pairwise interactions to be shrunk. Analyses were carried out using the glmnet package $[15,16]$.

Finally, survival-based classification trees were used to examine the possibility of higher-order interaction terms [17]. This approach first examines all possible dichotomous partitions of the data based on the 42 prognostic variables and chooses the one which best discriminates survival. These two corresponding partitions are then examined and split into subpartitions. The procedure continues recursively until a full tree is built. Crossvalidation was used to prune the tree by determining the number of partitions that minimizes reclassification error.

\section{Results}

\section{Distribution of Clinical, Lifestyle, and Tumor Expression Factors}

A total of 542 patients were included in the analysis, with 393 events, which included 260 episodes of cancer recurrence and an additional 133 deaths. The median recurrence-free survival was 2.1 years. The median followup time of those patients still alive at the time of this report was 7.4 years. The median follow-up time of those patients who had recurrent disease or who were dead was 1.4 years. Patient baseline characteristics and the 42 candidate prognostic variables are listed in table 1.

\section{Univariate and Multivariate Analyses}

In univariate analyses, after Bonferroni correction, patient age at cancer diagnosis, histology, cancer stage, cancer grade, presence of ascites, and presence of postoperative residual disease showed a statistically significant association with worse recurrence-free survival (table 1). No lifestyle factors, including oral contraceptive use, were associated with prognosis at $\mathrm{p}<0.05$. Overexpression of WT1 and p16 in tumors was also associated with worse recurrence-free survival. In contrast, overexpression of TFF3, MDM2, and p53 was associated with improved recurrence-free survival. Results using imputed data were similar (online suppl. tables 1 and 2; for all online suppl. material, see www. karger.com/doi/10.1159/000442710). 
Table 1. Distributions of patient characteristics and univariate associations with ovarian cancer

\begin{tabular}{|c|c|c|c|c|c|c|}
\hline Variable & Level & $\mathrm{n}$ & $\%$ & $\begin{array}{l}\text { Person- } \\
\text { years }\end{array}$ & HR (95\% CI) & $\mathrm{p}$ value \\
\hline \multicolumn{7}{|l|}{ Clinical factors } \\
\hline Age at cancer & $<50$ years & 96 & 65.63 & 373 & ref. & $<0.01$ \\
\hline \multirow[t]{3}{*}{ diagnosis } & $50-59$ years & 139 & 66.19 & 518 & $1.02(0.74-1.41)$ & \\
\hline & $60-69$ years & 149 & 71.14 & 509 & $1.15(0.84-1.57)$ & \\
\hline & $70+$ years & 158 & 83.54 & 422 & $1.62(1.20-2.19)$ & \\
\hline \multirow[t]{5}{*}{ Tumor histology } & Serous & 374 & 83.16 & 996 & ref. & $<0.01$ \\
\hline & Endometrioid & 91 & 41.76 & 499 & $0.30(0.21-0.42)$ & \\
\hline & Clear cell & 35 & 54.28 & 136 & $0.50(0.32-0.80)$ & \\
\hline & Mucinous & 18 & 33.33 & 110 & $0.22(0.10-0.49)$ & \\
\hline & Other & 24 & 79.17 & 82 & $0.80(0.51-1.28)$ & \\
\hline \multirow[t]{4}{*}{ Cancer stage } & 1 & 87 & 27.59 & 568 & ref. & $<0.01$ \\
\hline & 2 & 38 & 47.37 & 211 & $1.88(1.02-3.47)$ & \\
\hline & 3 & 328 & 81.40 & 886 & $5.85(3.84-8.91)$ & \\
\hline & 4 & 89 & 94.38 & 158 & $10.10(6.38-16.0)$ & \\
\hline \multirow[t]{2}{*}{ Grade } & Low & 91 & 37.36 & 534 & ref. & $<0.01$ \\
\hline & High & 449 & 79.73 & 1,276 & $3.57(2.51-5.09)$ & \\
\hline \multirow{2}{*}{$\begin{array}{l}\text { Ascites present at } \\
\text { surgery }\end{array}$} & No & 164 & 48.78 & 807 & ref. & $<0.01$ \\
\hline & Yes & 275 & 83.64 & 695 & $2.85(2.21-3.69)$ & \\
\hline \multirow{3}{*}{$\begin{array}{l}\text { Postoperative } \\
\text { residual disease }\end{array}$} & None & 238 & 51.26 & 1,137 & ref. & $<0.01$ \\
\hline & $\leq 1 \mathrm{~cm}$ & 152 & 92.11 & 308 & $3.52(2.74-4.52)$ & \\
\hline & $>1 \mathrm{~cm}$ & 70 & 91.43 & 107 & $4.80(3.51-6.55)$ & \\
\hline \multicolumn{7}{|c|}{ Lifestyle/reproductive factors } \\
\hline \multirow[t]{4}{*}{ Age at menarche } & $\leq 11$ years & 67 & 70.15 & 234 & ref. & 0.62 \\
\hline & 12 years & 114 & 70.18 & 402 & $1.01(0.71-1.45)$ & \\
\hline & 13 years & 122 & 66.39 & 446 & $0.89(0.62-1.28)$ & \\
\hline & $14+$ years & 113 & 74.34 & 370 & $1.10(0.77-1.57)$ & \\
\hline \multirow[t]{2}{*}{ Oral contraceptive use } & Never & 224 & 73.21 & 726 & ref. & 0.20 \\
\hline & Ever & 278 & 70.50 & 1,004 & $0.87(0.71-1.08)$ & \\
\hline \multirow[t]{5}{*}{ Parity/age at first birth } & Nulliparous & 90 & 68.89 & 344 & ref. & 0.32 \\
\hline & $1-2 / \leq 20$ years & 41 & 65.85 & 156 & $0.96(0.61-1.50)$ & \\
\hline & $1-2 />20$ years & 156 & 67.95 & 539 & $1.06(0.77-1.45)$ & \\
\hline & $3+/ \leq 20$ years & 94 & 73.40 & 300 & $1.25(0.89-1.77)$ & \\
\hline & $3+/>20$ years & 124 & 79.03 & 396 & $1.30(0.94-1.78)$ & \\
\hline \multirow[t]{3}{*}{ Education } & Less than high school & 32 & 68.75 & 119 & ref. & 0.70 \\
\hline & Finished high school & 160 & 73.75 & 549 & $1.18(0.75-1.87)$ & \\
\hline & More than high school & 280 & 70.71 & 958 & $1.10(0.71-1.71)$ & \\
\hline \multirow[t]{2}{*}{ Smoking status } & Never & 292 & 71.23 & 1,041 & ref. & 0.31 \\
\hline & Ever & 213 & 72.30 & 693 & $1.11(0.90-1.37)$ & \\
\hline
\end{tabular}


Table 1 (continued)

\begin{tabular}{|c|c|c|c|c|c|c|}
\hline Variable & Level & $\mathrm{n}$ & $\%$ & $\begin{array}{l}\text { Person- } \\
\text { years }\end{array}$ & $\mathrm{HR}(95 \% \mathrm{CI})$ & $\mathrm{p}$ value \\
\hline \multicolumn{7}{|c|}{ Tumor protein expression } \\
\hline \multirow{2}{*}{ ARID1A } & $<1-50 \%$ positive & 55 & 58.18 & 252 & ref. & 0.02 \\
\hline & $>50 \%$ positive & 449 & 74.16 & 1,457 & $1.56(1.08-2.24)$ & \\
\hline \multirow[t]{3}{*}{$\beta 2$ defensin } & None & 20 & 85.00 & 48 & ref. & 0.08 \\
\hline & Weak & 185 & 77.30 & 651 & $0.68(0.41-1.13)$ & \\
\hline & Moderate/strong & 264 & 69.32 & 877 & $0.59(0.36-0.97)$ & \\
\hline \multirow[t]{3}{*}{ CD68 } & None/weak & 138 & 68.12 & 523 & ref. & 0.14 \\
\hline & Moderate & 232 & 75.43 & 760 & $1.23(0.96-1.58)$ & \\
\hline & Strong & 87 & 77.01 & 259 & $1.34(0.98-1.84)$ & \\
\hline \multirow[t]{3}{*}{ CD8 } & None/weak & 317 & 73.50 & 1,043 & ref. & 0.10 \\
\hline & Moderate & 117 & 74.36 & 388 & $0.98(0.77-1.25)$ & \\
\hline & Strong & 42 & 59.52 & 185 & $0.63(0.42-0.97)$ & \\
\hline \multirow[t]{3}{*}{ DKK1 } & Negative $(<1 \%)$ & 368 & 75.00 & 1,169 & ref. & $<0.01$ \\
\hline & $1-50 \%$ positive & 98 & 75.51 & 331 & $0.99(0.76-1.28)$ & \\
\hline & $>50 \%$ positive & 24 & 41.67 & 135 & $0.34(0.17-0.66)$ & \\
\hline \multirow[t]{3}{*}{ ER } & Negative $(<1 \%)$ & 123 & 73.17 & 391 & ref. & 0.04 \\
\hline & $1-50 \%$ positive & 91 & 84.62 & 267 & $1.21(0.89-1.65)$ & \\
\hline & $>50 \%$ positive & 286 & 68.88 & 1,034 & $0.87(0.67-1.12)$ & \\
\hline \multirow[t]{2}{*}{ Fibrinogen } & None & 425 & 73.65 & 1,423 & ref. & 0.89 \\
\hline & Weak/moderate/strong & 38 & 73.68 & 119 & $1.03(0.70-1.51)$ & \\
\hline \multirow[t]{4}{*}{ FOLR1 } & Negative $(<1 \%)$ & 88 & 59.09 & 373 & ref. & 0.04 \\
\hline & Weak cyto., membr. & 189 & 73.02 & 670 & $1.29(0.94-1.78)$ & \\
\hline & Moderate/strong cyto., & 159 & 79.25 & 461 & $1.59(1.15-2.21)$ & \\
\hline & $\begin{array}{l}\text { heterogeneous membr. } \\
\text { Full diffuse cyto., consis- } \\
\text { tent strong membr. }\end{array}$ & 69 & 78.26 & 192 & $1.45(0.98-2.14)$ & \\
\hline \multirow[t]{3}{*}{ gp96 } & None & 16 & 93.75 & 38 & ref. & 0.06 \\
\hline & Weak & 153 & 76.47 & 501 & $0.69(0.41-1.19)$ & \\
\hline & Moderate/strong & 320 & 68.75 & 1,135 & $0.58(0.35-0.98)$ & \\
\hline \multirow[t]{2}{*}{ Heparan sulfate } & None/weak & 198 & 67.17 & 707 & ref. & 0.13 \\
\hline & Moderate/strong & 268 & 77.24 & 864 & $1.18(0.95-1.47)$ & \\
\hline \multirow[t]{3}{*}{ Heparanase } & None & 170 & 77.06 & 553 & ref. & 0.23 \\
\hline & Weak & 239 & 70.71 & 810 & $0.82(0.65-1.04)$ & \\
\hline & Moderate/strong & 50 & 72.00 & 181 & $0.83(0.57-1.20)$ & \\
\hline \multirow{3}{*}{$\begin{array}{l}\text { High-mobility } \\
\text { group } \\
\text { box } 1\end{array}$} & None & 154 & 77.92 & 519 & ref. & 0.10 \\
\hline & Weak & 265 & 68.30 & 920 & $0.80(0.64-1.01)$ & \\
\hline & Moderate/strong & 42 & 83.33 & 127 & $1.07(0.73-1.55)$ & \\
\hline \multirow[t]{3}{*}{ HNF1B } & Negative $(<1 \%)$ & 384 & 76.82 & 1,178 & ref. & $<0.01$ \\
\hline & $1-50 \%$ positive & 61 & 62.30 & 252 & $0.70(0.50-0.98)$ & \\
\hline & $>50 \%$ positive & 55 & 58.18 & 245 & $0.61(0.42-0.87)$ & \\
\hline \multirow[t]{2}{*}{ hsp60 } & Weak & 122 & 67.21 & 483 & ref. & 0.09 \\
\hline & Moderate/strong & 363 & 75.21 & 1,143 & $1.24(0.96-1.59)$ & \\
\hline \multirow[t]{3}{*}{ hsp70 } & Low & 164 & 64.63 & 638 & ref. & $<0.01$ \\
\hline & Medium & 181 & 81.22 & 516 & $1.51(1.18-1.94)$ & \\
\hline & High & 158 & 71.52 & 542 & $1.12(0.86-1.46)$ & \\
\hline
\end{tabular}


Table 1 (continued)

\begin{tabular}{|c|c|c|c|c|c|c|}
\hline Variable & Level & $\mathrm{n}$ & $\%$ & $\begin{array}{l}\text { Person- } \\
\text { years }\end{array}$ & HR (95\% CI) & $\mathrm{p}$ value \\
\hline $\mathrm{I} \kappa \mathrm{B} a$ & $\begin{array}{l}\text { None } \\
\text { Weak } \\
\text { Moderate/strong }\end{array}$ & $\begin{array}{l}128 \\
248 \\
106\end{array}$ & $\begin{array}{l}78.91 \\
72.18 \\
68.87\end{array}$ & $\begin{array}{l}423 \\
852 \\
346\end{array}$ & $\begin{array}{l}\text { ref. } \\
0.82(0.65-1.05) \\
0.81(0.60-1.09)\end{array}$ & 0.24 \\
\hline I $\kappa \mathrm{K} \beta$ & $\begin{array}{l}\text { None } \\
\text { Weak } \\
\text { Moderate/strong }\end{array}$ & $\begin{array}{r}97 \\
268 \\
109\end{array}$ & $\begin{array}{l}77.32 \\
70.15 \\
72.48\end{array}$ & $\begin{array}{l}334 \\
935 \\
346\end{array}$ & $\begin{array}{l}\text { ref. } \\
0.85(0.65-1.11) \\
0.92(0.67-1.26)\end{array}$ & 0.49 \\
\hline MDM2 & $\begin{array}{l}\text { Negative }(<1 \%) \\
\geq 1 \% \text { positive }\end{array}$ & $\begin{array}{l}340 \\
152\end{array}$ & $\begin{array}{l}79.71 \\
58.55\end{array}$ & $\begin{array}{l}982 \\
658\end{array}$ & $\begin{array}{l}\text { ref. } \\
0.58(0.45-0.74)\end{array}$ & $<0.01$ \\
\hline MMP9 & $\begin{array}{l}\text { None } \\
\text { Weak } \\
\text { Moderate/strong }\end{array}$ & $\begin{array}{r}177 \\
259 \\
28\end{array}$ & $\begin{array}{l}79.66 \\
68.73 \\
75.00\end{array}$ & $\begin{array}{l}579 \\
888 \\
103\end{array}$ & $\begin{array}{l}\text { ref. } \\
0.77(0.62-0.97) \\
0.76(0.48-1.20)\end{array}$ & 0.07 \\
\hline MyD88 & $\begin{array}{l}\text { None/weak } \\
\text { Moderate } \\
\text { Strong }\end{array}$ & $\begin{array}{r}96 \\
209 \\
159\end{array}$ & $\begin{array}{l}59.38 \\
73.68 \\
78.62\end{array}$ & $\begin{array}{l}405 \\
691 \\
480\end{array}$ & $\begin{array}{l}\text { ref. } \\
1.46(1.07-1.98) \\
1.62(1.18-2.23)\end{array}$ & 0.01 \\
\hline p16 & $\begin{array}{l}\text { Negative }(<1 \%) \\
1-50 \% \text { positive } \\
>50 \% \text { positive }\end{array}$ & $\begin{array}{r}25 \\
242 \\
232\end{array}$ & $\begin{array}{l}56.00 \\
67.36 \\
80.17\end{array}$ & $\begin{array}{l}103 \\
951 \\
630\end{array}$ & $\begin{array}{l}\text { ref. } \\
1.27(0.73-2.19) \\
1.89(1.09-3.25)\end{array}$ & $<0.01$ \\
\hline p50 & $\begin{array}{l}\text { None } \\
\text { Weak } \\
\text { Moderate/strong }\end{array}$ & $\begin{array}{r}77 \\
240 \\
142\end{array}$ & $\begin{array}{l}84.42 \\
75.00 \\
64.79\end{array}$ & $\begin{array}{l}238 \\
779 \\
525\end{array}$ & $\begin{array}{l}\text { ref. } \\
0.84(0.64-1.12) \\
0.64(0.47-0.89)\end{array}$ & 0.02 \\
\hline p53 & $\begin{array}{l}\text { Negative }(<1 \%) \\
1-50 \% \text { positive } \\
>50 \% \text { positive }\end{array}$ & $\begin{array}{r}99 \\
166 \\
230\end{array}$ & $\begin{array}{l}78.79 \\
54.82 \\
84.78\end{array}$ & $\begin{array}{l}289 \\
761 \\
606\end{array}$ & $\begin{array}{l}\text { ref. } \\
0.52(0.38-0.70) \\
1.12(0.86-1.46)\end{array}$ & $<0.01$ \\
\hline p65 & $\begin{array}{l}\text { None/weak } \\
\text { Moderate/strong }\end{array}$ & $\begin{array}{r}40 \\
452\end{array}$ & $\begin{array}{l}65.00 \\
73.45\end{array}$ & $\begin{array}{r}163 \\
1,491\end{array}$ & $\begin{array}{l}\text { ref. } \\
1.35(0.90-2.03)\end{array}$ & 0.15 \\
\hline Phospho-IкB $\alpha$ & $\begin{array}{l}\text { None } \\
\text { Weak/moderate/strong }\end{array}$ & $\begin{array}{l}216 \\
261\end{array}$ & $\begin{array}{l}76.39 \\
68.97\end{array}$ & $\begin{array}{l}729 \\
899\end{array}$ & $\begin{array}{l}\text { ref. } \\
0.87(0.70-1.07)\end{array}$ & 0.19 \\
\hline Phospho-p65 & $\begin{array}{l}\text { None } \\
\text { Weak } \\
\text { Moderate/strong }\end{array}$ & $\begin{array}{r}50 \\
297 \\
134\end{array}$ & $\begin{array}{l}80.00 \\
71.72 \\
72.39\end{array}$ & $\begin{array}{r}172 \\
1,033 \\
427\end{array}$ & $\begin{array}{l}\text { ref. } \\
0.85(0.61-1.20) \\
0.88(0.61-1.27)\end{array}$ & 0.66 \\
\hline PR & $\begin{array}{l}\text { Negative }(<1 \%) \\
1-50 \% \text { positive } \\
>50 \% \text { positive }\end{array}$ & $\begin{array}{r}297 \\
116 \\
77\end{array}$ & $\begin{array}{l}79.80 \\
75.00 \\
48.05\end{array}$ & $\begin{array}{l}827 \\
417 \\
383\end{array}$ & $\begin{array}{l}\text { ref. } \\
0.79(0.61-1.00) \\
0.40(0.28-0.56)\end{array}$ & $<0.01$ \\
\hline TFF3 & $\begin{array}{l}\text { Negative }(<1 \%) \\
1-50 \% \text { positive } \\
>50 \% \text { positive }\end{array}$ & $\begin{array}{r}327 \\
105 \\
62\end{array}$ & $\begin{array}{l}81.04 \\
70.48 \\
40.32\end{array}$ & $\begin{array}{l}951 \\
364 \\
347\end{array}$ & $\begin{array}{l}\text { ref. } \\
0.82(0.63-1.06) \\
0.32(0.21-0.48)\end{array}$ & $<0.01$ \\
\hline TLR4 & $\begin{array}{l}\text { None/weak } \\
\text { Moderate } \\
\text { Strong }\end{array}$ & $\begin{array}{r}164 \\
215 \\
90\end{array}$ & $\begin{array}{l}65.85 \\
74.42 \\
80.00\end{array}$ & $\begin{array}{l}633 \\
731 \\
236\end{array}$ & $\begin{array}{l}\text { ref. } \\
1.23(0.96-1.57) \\
1.60(1.19-2.16)\end{array}$ & $<0.01$ \\
\hline Vimentin & $\begin{array}{l}\text { Negative }(<1 \%) \\
1-50 \% \text { positive } \\
>50 \% \text { positive }\end{array}$ & $\begin{array}{r}354 \\
93 \\
44\end{array}$ & $\begin{array}{l}78.25 \\
61.29 \\
65.91\end{array}$ & $\begin{array}{r}1,081 \\
384 \\
166\end{array}$ & $\begin{array}{l}\text { ref. } \\
0.63(0.47-0.83) \\
0.65(0.44-0.97)\end{array}$ & $<0.01$ \\
\hline WT1 & $\begin{array}{l}\text { Negative }(<1 \%) \\
1-50 \% \text { positive } \\
>50 \% \text { positive }\end{array}$ & $\begin{array}{r}141 \\
79 \\
283\end{array}$ & $\begin{array}{l}51.77 \\
78.48 \\
82.69\end{array}$ & $\begin{array}{l}681 \\
269 \\
736\end{array}$ & $\begin{array}{l}\text { ref. } \\
1.98(1.40-2.78) \\
2.48(1.89-3.24)\end{array}$ & $<0.01$ \\
\hline
\end{tabular}

$\mathrm{HR}=$ Hazard ratio; ref. $=$ reference; memb. = membrane staining; cyto. = cytoplasmic staining. 
Table 2. Multivariate associations of clinical and tumor expression variables with ovarian cancer recurrence-free survival

\begin{tabular}{|c|c|c|c|c|c|}
\hline Variable & Level & $\mathrm{n}$ & $\%$ & $\begin{array}{l}\text { Person- } \\
\text { years }\end{array}$ & HR (95\% CI) \\
\hline \multicolumn{6}{|l|}{ Clinical factors } \\
\hline \multirow[t]{4}{*}{ Age at cancer diagnosis } & $<50$ years & 96 & 65.63 & 373 & ref. \\
\hline & $50-59$ years & 139 & 66.19 & 518 & $0.99(0.71-1.38)$ \\
\hline & $60-69$ years & 149 & 71.14 & 509 & $0.81(0.58-1.12)$ \\
\hline & $70+$ years & 158 & 83.54 & 422 & $1.31(0.95-1.79)$ \\
\hline \multirow[t]{5}{*}{ Tumor histology } & Serous & 374 & 83.16 & 996 & ref. \\
\hline & Endometrioid & 91 & 41.76 & 499 & $1.14(0.44-2.93)$ \\
\hline & Clear cell & 35 & 54.28 & 136 & $0.81(0.54-1.2)$ \\
\hline & Mucinous & 18 & 33.33 & 110 & $1.14(0.69-1.89)$ \\
\hline & Other & 24 & 79.17 & 82 & $1.27(0.79-2.05)$ \\
\hline \multirow[t]{4}{*}{ Cancer stage } & 1 & 87 & 27.59 & 568 & ref. \\
\hline & 2 & 38 & 47.37 & 211 & $1.72(0.9-3.3)$ \\
\hline & 3 & 328 & 81.40 & 886 & $3.18(1.86-5.44)$ \\
\hline & 4 & 89 & 94.38 & 158 & $4.77(2.69-8.46)$ \\
\hline \multirow[t]{2}{*}{ Grade } & Low & 91 & 37.36 & 534 & ref. \\
\hline & High & 449 & 79.73 & 1,276 & $1.28(0.84-1.96)$ \\
\hline \multirow[t]{2}{*}{ Ascites present at surgery } & No & 164 & 48.78 & 807 & ref. \\
\hline & Yes & 275 & 83.64 & 695 & $1.47(1.13-1.91)$ \\
\hline \multirow[t]{3}{*}{ Postoperative residual disease } & None & 238 & 51.26 & 1,137 & ref. \\
\hline & $\leq 1 \mathrm{~cm}$ & 152 & 92.11 & 308 & $1.54(1.16-2.04)$ \\
\hline & $>1 \mathrm{~cm}$ & 70 & 91.43 & 107 & $2.52(1.79-3.54)$ \\
\hline \multicolumn{6}{|l|}{ Tumor protein expression } \\
\hline \multirow[t]{3}{*}{$\mathrm{PR}$} & Negative $(<1 \%)$ & 297 & 79.80 & 827 & ref. \\
\hline & $1-50 \%$ positive & 116 & 75.00 & 417 & $0.92(0.71-1.21)$ \\
\hline & $>50 \%$ positive & 77 & 48.05 & 383 & $0.64(0.44-0.94)$ \\
\hline \multirow[t]{3}{*}{ TFF3 } & Negative $(<1 \%)$ & 327 & 81.04 & 951 & ref. \\
\hline & $1-50 \%$ positive & 105 & 70.48 & 364 & $1.36(1.03-1.81)$ \\
\hline & $>50 \%$ positive & 62 & 40.32 & 347 & $0.84(0.53-1.33)$ \\
\hline
\end{tabular}

Tumor expression variables were chosen using GL Cox proportional hazards regression analysis. Clinical variables (patient age at cancer diagnosis, histology, stage, grade, ascites, and extent of postoperative residual disease) were included in the final multivariate model regardless of LASSO results. HR and CI estimated using Cox regression of multiple imputed data adjusted for all variables are shown. HR = Hazard ratio; ref. = reference.

In Cox regression multivariate analyses, patient age at cancer diagnosis, tumor grade, and presence of ascites were marginally associated with prognosis, while tumor histology was not significantly associated with cancer recurrence (table 2). Overexpression of PR appeared to be a protective prognostic variable in multivariate Cox regression models [hazard ratio for $>50 \%$ of cells positive, 0.64 (95\% CI $0.44-0.94$ ) compared to $<1 \%$ positive]. Associations with TFF3 were more complex: women with moderate expression were at a marginally increased risk of recurrence, and women with high expression were at a similar to slightly lower risk, compared to women with no expression.

\section{Potential Interactions of Variables}

In traditional analyses of all possible pairwise interactions, pairs $(\mathrm{p}<0.001)$ that were associated with recurrence-free survival included histology with extent of post- 
Table 3. Pairwise interactions $(\mathrm{p}<0.001)$ and association with ovarian cancer recurrence-free survival

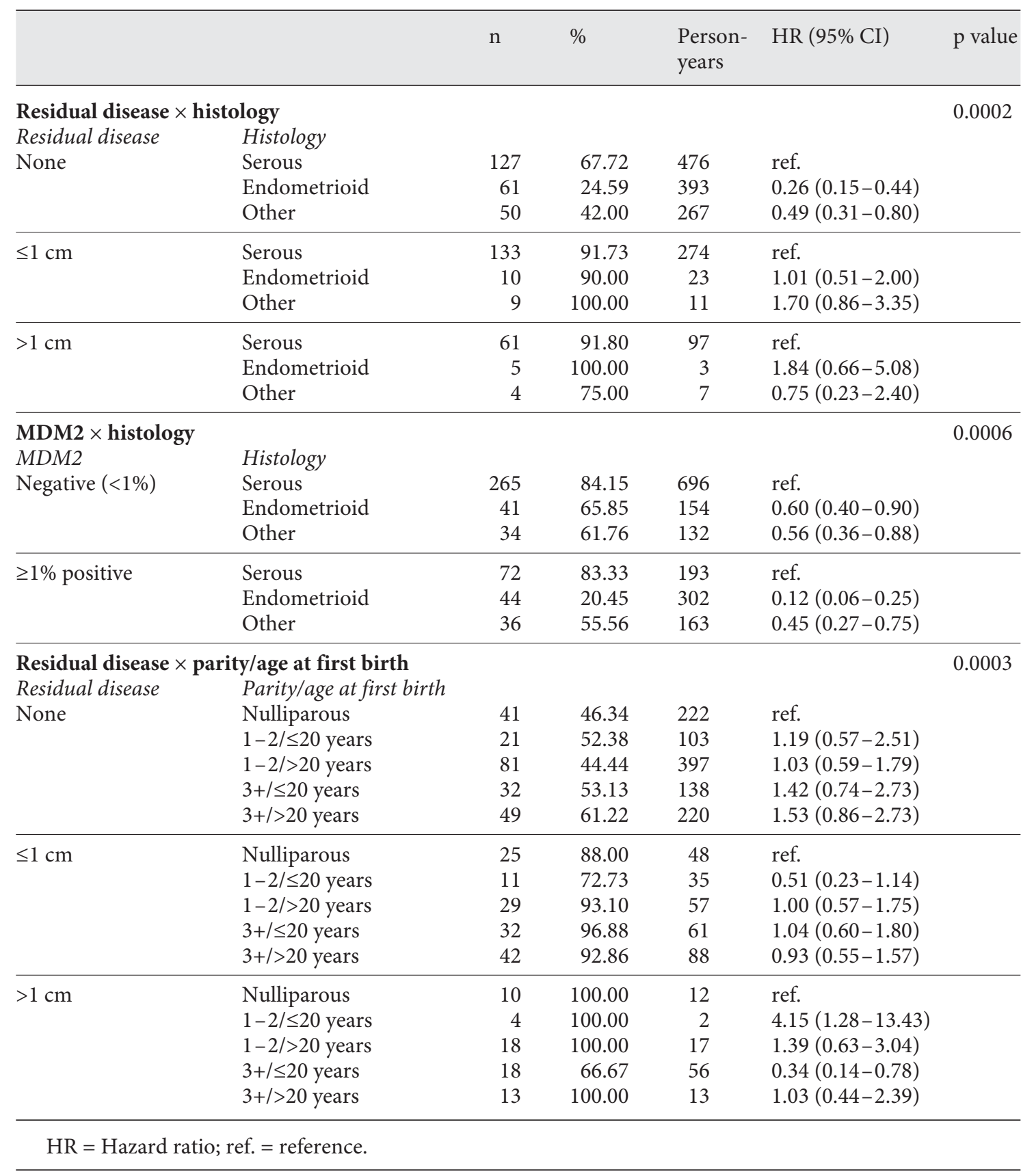

operative residual disease, parity/age at first birth with extent of postoperative residual disease, and histology with MDM2 (table 3). These findings suggest that nonserous histology is most protective among women with no residual disease or with positive MDM2 expression, and that increased parity may be detrimental among women with no residual disease. However, none of these prog- nostic interactions remained statistically significant after accounting for multiple testing.

Using elastic net methodology, we observed null results with no single interaction remaining in the final model for more than one of the five imputed datasets for any of the penalty levels tested. Both approaches (shrinking only interaction terms or the two-step approach) re- 
sulted in the same conclusions. Crossvalidation analyses with a survival-based classification tree suggested an optimal tree size of one node; no single data split, let alone combination of splits, dramatically improved the discrimination of recurrence-free survival.

\section{Discussion}

This study examined the prognostic significance of numerous clinical and tumor protein expression variables and their potential interactions in ovarian cancer patients. As expected, we observed that cancer stage, ascites, postoperative residual disease, and overexpression of PR were associated with worse recurrence-free survival. In addition, this study revealed two unexpected findings. First, using both traditional and contemporary analysis methods, we found limited pairwise or higherorder interactions with respect to ovarian cancer recurrence-free survival among the 42 clinical, lifestyle, and tumor expression factors examined. After adjusting for multiple testing, using the elastic net method, and confirming findings by means of a survival-based classification tree, we found no evidence of interactions between any of these variables. Some interactions may merit consideration in larger studies. Nonetheless, this study provides important precedent for assessing interactions among multiple, diverse groups of variables in an effort to better understand ovarian cancer prognosis.

Interestingly, the elastic net analysis methodology used in this study is timely in view of numerous ongoing studies that seek to examine interactions between an expansive number of variables - particularly genomic factors - and their associations with respect to cancer risk. Although the current study did not include genomic data, other investigations may use similar methodology when analyzing large datasets to demonstrate the complexity of genetic factors or epistasis. Future research might use similar methodology to examine putative in- teractions among a variety of factors associated with recurrence-free survival, including genomic ones. Thus, we view our findings as an invitation to further explore potential interactions.

The second important finding of our paper centers on TFF3 expression, a marker that appears to have protective effects on epithelial cells and that has been relatively understudied in ovarian cancer; it was associated with a variable but statistically significant risk of cancer recurrence based on the extent of expression. This protein has begun to receive increasing attention in gastrointestinal cancers as a potential marker for gastric cancer screening and, therefore, may merit further study in ovarian cancer $[18,19]$. Further validation of this incidental finding in conjunction with confirmation of effect sizes might lead to further understanding of the clinical relevance of this preliminary observation.

This study has both strengths and limitations. One limitation is that although our sample size appears robust by some standards, the number of variables introduced into our models ultimately led to diminished power as a result of the multiple testing burden. Despite this limitation, our efforts to champion the elastic net methodology with the inclusion of diverse groups of variables may prompt others to undertake a similar approach within larger multi-institutional datasets. Another limitation is that for certain relevant variables, such as BRCA1 and 2 mutation status, we had too little data to incorporate into our models. In terms of strengths, we believe again that the application of the elastic net methodology to such a diverse group of variables, with the goal of better understanding the prognosis of ovarian cancer patients, is unique and merits more widespread use.

\section{Acknowledgement}

This work was funded by the Minnesota Ovarian Cancer Alliance, R01-CA122443, P30-CA15083, and P50-CA136393.
References

Prognostic Factors in Ovarian Cancer
1 Siegel R, Ma J, Zou Z, Jemal A: Cancer statistics 2014. CA Cancer J Clin 2014;64:9-29.

-2 Kelly MG, Alvero AB, Chen R, et al: TLR-4 signaling promotes tumor growth and paclitaxel chemoresistance in ovarian cancer. Cancer Res 2006;66:3859-3868.

-3 Wang AC, Ma YB, Wu FX, et al: TLR4 induces tumor growth and inhibits paclitaxel activity in MyD88-positive human ovarian carcinoma. Oncol Lett 2014;7:871-877.
4 Gram IT, Lukanova A, Brill I, et al: Cigarette smoking and risk of histologic subtypes of epithelial ovarian cancer in the EPIC cohort. Int J Cancer 2012;130:2204-2210.

5 Kjaerbye-Thygesen A, Frederiksen K, Hogdall EV, et al: Do risk factors for epithelial ovarian cancer have an impact on prognosis? Focus on previous pelvic surgery and reproductive variables. Eur J Gynaecol Oncol 2006; 27:467-472. 
6 Wahner Hendrickson AE, Hawthorne KM, Goode EL, et al: Assessment of published models and prognostic variables in epithelial ovarian cancer at Mayo Clinic. Gynecol Oncol 2015;137:77-85.

7 Kobel M, Kalloger SE, Lee S, et al: Biomarkerbased ovarian carcinoma typing: a histologic investigation in the ovarian tumor tissue analysis consortium. Cancer Epidemiol Biomarkers Prev 2013;22:1677-1686.

$\checkmark 8$ Köbel M, Madore J, Ramus SJ, Clarke BA, Pharoah PDP, Deen S, et al: Evidence for a time-dependent association between FOLR1 expression and survival from ovarian carcinoma: implications for clinical testing. An Ovarian Tumor Tissue Analysis consortium study. Br J Cancer 2014;111:2297-2307.

-9 Sieh W, Kobel M, Longacre TA, et al: Hormone receptor expression and ovarian cancer survival: an Ovarian Tumor Tissue Analysis consortium study. Lancet Oncol 2013;14: 853-862.
Shen H, Fridley BL, Song H, et al: Epigenetic analysis leads to identification of HNF1B as a subtype-specific susceptibility gene for ovarian cancer. Nat Commun 2013;4:1628.

11 Rubin DB, Schenker N: Multiple imputation for interval estimation from simple random samples with ignorable nonresponse. J Am Stat Assoc 1986;81:366-374.

12 Simon N, Friedman J, Hastie T, Tibshirani R: A sparse-Group LASSO. J Comp Graph Stat 2013;22:231-245.

13 Tibshirani R: Regression shrinkage and selection via the lasso: a retrospective. J R Stat Soc B 2011;73:273-282.

14 Zou H, Hastie T: Regularization and variable selection via the elastic net. J R Stat Soc B 2005; 67:301-320.
15 Friedman J, Hastie T, Tibshirani R: Regularization paths for generalized linear models via coordinate descent. J Stat Softw 2010;33:1-22.

16 Simon N, Friedman J, Hastie T, Tibshirani R: Regulation paths for Cox's proportional hazards model via coordinate descent. J Stat Softw 2011;39:1-13.

17 Therneau TM, Atkinson EJ: An Introduction to Recursive Partitioning Using the RPART Routines. Technical Report Series 61. Rochester, Department of Health Science Research, Mayo Clinic, 1997.

18 Ding A, Zhao W, Shi X, et al: Impact of NPM, TFF3 and TACC1 on the prognosis of patients with primary gastric cancer. PLoS One 2013;8:e82136.

19 Huang Z, Zhang X, Lu H, et al: Serum trefoil factor is a promising non-invasive biomarker for gastric cancer screening: a monocentric cohort study in China. BMC Gastroenterol $2014 ; 14: 74$. 